\title{
超声振动改善电铸表面粗糙度的机理*
}

\author{
杨光胡志超 张军皮钧刘中生 \\ (集美大学机械与能源工程学院 厦门 361021)
}

\begin{abstract}
摘要: 探讨超声振动改善电铸表面粗粘度的机理。试验表明, 阴极板的超声振动能明显改善电铸层表面的粗樯度。对其机理, 归纳为三点: (1) 阴极板的超声振动使得电铸液在其边界层(阴极板附近的一个电铸液薄层)内产生众多的小浴流, 其旋转方向 以超声频率交替更换, 这就使得镍离子浓度(垂直于电铸表面方向)在边界层内更趋于均匀化, 提高了镍离子在边界层内的有 效扩散系数; (2) 阴极板边界层内更加均匀的镍离子浓度分布, 抑制了肌瘤和枝晶的生长; (3) 阴极表面生成的氢气核, 在其 初级阶段, 被超声微射流驱除, 无法继续生长, 从而使微小气孔等缺陷得到了抑制, 同时, 也降低了氢离子在阴极析出的反 应速率, 改善了电流效率。阴极板的固有振动模态计算、阴极板边界层的流场模拟、镍离子扩散系数的计算数据、电铸表面 粗粘度测量和电子显微镜图像, 都支持这个机理。
\end{abstract}

关键词: 电铸; 超声振动; 机理; 粗糙度; 扩散; 结晶

中图分类号: $\mathrm{TQ} 153$

\section{Mechanism of Improved Surface Roughness in Electroforming Assisted by Ultrasonic Vibration}

\author{
YANG Guang HU Zhichao ZHANG Jun PI Jun LIU Zhongsheng \\ (College of Mechanical and Energy Engineering, Jimei University, Xiamen 361021)
}

\begin{abstract}
A mechanism is proposed that can explain the improvement of surface roughness in electroforming assisted by ultrasonic vibration. Experiments have shown that ultrasonic vibration, in particular, ultrasonic vibration from a cathode plate can significantly improve surface roughness in electroforming. Building upon our experiments, the mechanism is summarized as the following three points. (1) Ultrasonic vibration from the cathode plate produces numerous eddies in the boundary layer on the top of the cathode plate, alternating the flow directions of eddies at an ultrasonic frequency, and as a consequence, the electrolyte in the boundary layer is well mixed leading to evener distributions of the concentration of nickel ions. In another words, the effective diffusion coefficient of nickel ions in the electrolyte is increased by ultrasonic vibration. (2) The evener distributions of the concentration of nickel ions suppress the growth of dendrites and bumps. (3) Ultrasonic microjet from the cathode plate can drive tiny nuclei of hydrogen gas at the initial stage of their formation inhibiting the continuing growth of tiny gas (needle) hole. In this way, the rate of hydrogen production is decreased with the efficiency of electric current improved. The mechanism is supported by the calculation of the natural vibration modes of the cathode plate, the numerical simulation of the flow field of electrolyte in the boundary layer, the numerical simulation of the effective coefficient of nickel ions in the electrolyte, and the measurement of surface roughness and the SEM images of the surfaces fabricated by the electroforming.
\end{abstract}

Key words: electroforming; ultrasonic vibration; mechanisms; surface roughness; diffusion; crystallization

\section{0 前言}

电铸作为金属零件制造的电化学方法，在机械 制造业有广泛的应用, 塑铸用的模具通常是由电铸

* 国家自然科学基金(51175225)、福建省科技重点(2017H0025)和福建省自 然科学基金(2017J01700)资助项目。20180501 收到初稿, 20181119 收到 修改稿
法复制原始金属模具得到的 ${ }^{[1-2]}$ 。对于复制几何形状 复杂的模具, 例如, 微棱雉阵列反光材料(能按原路 反射入射光，用于高速公路路标等场合)的电铸模， 要求每个微棱雉电铸表面粗粘度小于 $20 \mathrm{~nm}$, 从而 保证产品的反光性能 ${ }^{[3]}$ 。电铸微结构表面粗䊁度难 于控制，不仅仅是因为微结构有较多的凸凹边角， 导致电流分布不均匀 ${ }^{[4-6]}$, 也因为电沉积过程往往伴 随着肌瘤、枝晶的生长和氢气孔的产生。针对上述 
问题, 一个典型方法是将超声振动引入到电沉积过 程中 ${ }^{[7-13]}$ 。例如, LIU 等 ${ }^{[11]}$ 采用超声摚拌方法对铜 基板进行了 $\mathrm{Ni}-\mathrm{SiC}$ 纳米复合涂层的制备, 获得了孔 隙度低和结晶细化的沉积表面; 明平美等 ${ }^{[12]}$ 所做的 超声振动辅助电铸镍微器件, 积瘤和氢气孔等缺陷 明显减少。

本文作者的试验也证实了超声振动辅助电铸会 抑制肌瘤和枝晶的生长以及抑制氢气孔的出现, 从 而降低表面粗粘度。但是, 关于超声振动降低表面 粗粘度的机理, 报道得不多。本文试图将超声振动 辅助电铸降低表面粗粘度的机理向前推进一步, 为 超精密电铸(几十甚至几个纳米粗粘度)的发展打下 坚实的理论基础。

\section{1 超声振动辅助电铸与机械搅拌辅助 电铸的粗糙度对比}

将超声振动引入到电铸过程中, 有多个途径: 一个途径是振动通过超声清洗槽中的水传递给电 铸槽壁面, 再经过电铸槽壁面传递给电铸液 ${ }^{[14-16]}$; 另一个途径是将变幅杆的输出端插入到电铸液中 施加超声振动 ${ }^{[17-18]}$ 。本文的试验是将超声振动施加 在阴极板上, 激励出阴极板的高频振动, 通过阴极 板, 将超声振动作用到电铸液中, 尤其是作用到阴 极板附近的电铸液中。本文侧重于探讨这类阴极板 本身的超声振动对铸层表面粗粘度的改善效果和 机理。

超声振动辅助电铸可以降低表面粗糙度, 解释 这个现象要求揭示超声振动是如何影响镍离子的沉 积过程的, 这个过程包含电化学反应和镍离子结晶 过程。对于机械搅拌辅助电铸而言, 电铸液在边界 层的流场有比较急剧的变化, 在阴极板上, 其速度 为零, 镍离子浓度很低; 稍微离开阴极板表面, 流 动速度会有很大增长, 镍离子浓度也有较大增长; 再进一步远离阴极板表面, 镍离子浓度的变化比较 平缓。对于阴极板超声振动辅助电铸而言, 情况有 很大的不同。

\section{1 试验装置及参数}

电铸装置结构如图 1 所示。超声换能器将电 能转换为机械能, 机械振动经变幅杆放大之后, 由变幅杆前端垂直作用在阴极板上, 使阴极板产 生高频弯曲振动。阳极镍板的纯度大于 $99.9 \%$, 阴 极 (反光材料)原始模具微棱镜镍板)的有效尺寸为 7 $\mathrm{cm} \times 7 \mathrm{~cm} \times 2 \mathrm{~mm}$ 。阳极与阴极均铅直放置, 极板间 距为 $15 \mathrm{~cm}$, 使用直流电源, 电铸液 $\mathrm{pH}$ 值为 4.2 ,
温度为 $50{ }^{\circ} \mathrm{C}$, 电流密度为 $5 \mathrm{~A} \cdot \mathrm{dm}^{-2}$, 机械搅拌速 度为 $1000 \mathrm{r} / \mathrm{min}$, 超声频率为 $40 \mathrm{kHz}$, 功率为 300 $\mathrm{W}$ 和 $400 \mathrm{~W}$ 。阴极超声振动辅助电铸与机械摚拌 辅助电铸之前, 阴极模板均进行相同的前处理: 用丙酮和 $10 \%$ 的稀盐酸溶液对其进行化学去脂和 活化处理。所用电铸液为 $35 \mathrm{~g} / \mathrm{L}$ 硼酸、 $50 \mathrm{~g} / \mathrm{L}$ 氯 化镍、 $280 \mathrm{~g} / \mathrm{L}$ 硫酸镍和 $0.07 \mathrm{~g} / \mathrm{L}$ 十二烷基磺酸钠 的混合水溶液。

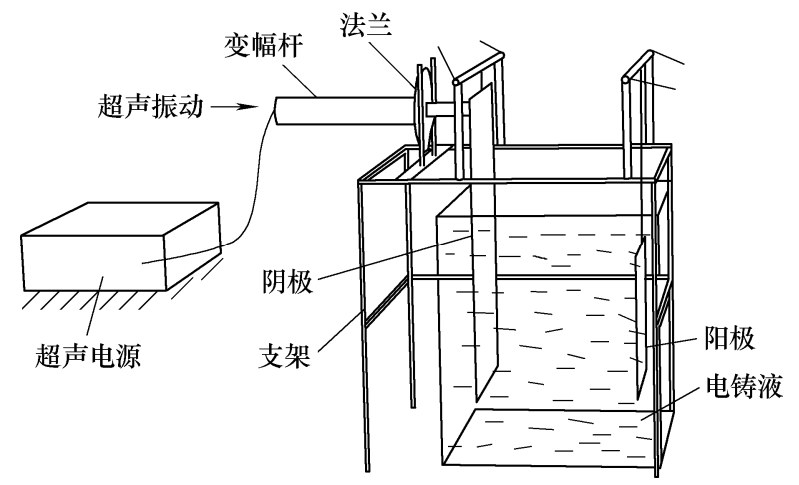

图 1 阴极超声振动电沉积装置结构图

\section{2 粗糙度和电镜图像}

经 5 小时电沉积后, 得到厚度为 $0.6 \mathrm{~mm}$ 厚的铸 层。图 $2 \mathrm{a} 、 2 \mathrm{~b}$ 和 $2 \mathrm{c}$ 分别是常规机械摚拌和阴极板 超声振动下沉积层的三维形貌及粗粘度。从图 2 可 以看出: 机械搅拌的沉积表面不平, 含有较多的气 孔; 超声振动辅助电铸的沉积层表面均匀, 少有气 孔等缺陷。

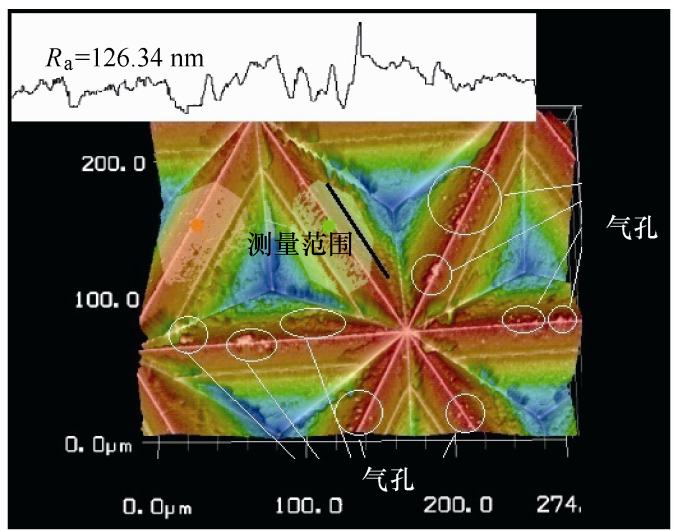

(a) 机械摚拌

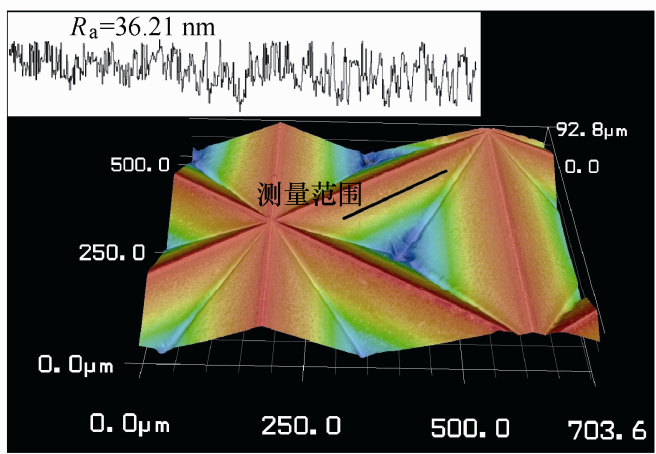

(b) 阴极超声振动 $(40 \mathrm{kHz}, 400 \mathrm{~W})$ 


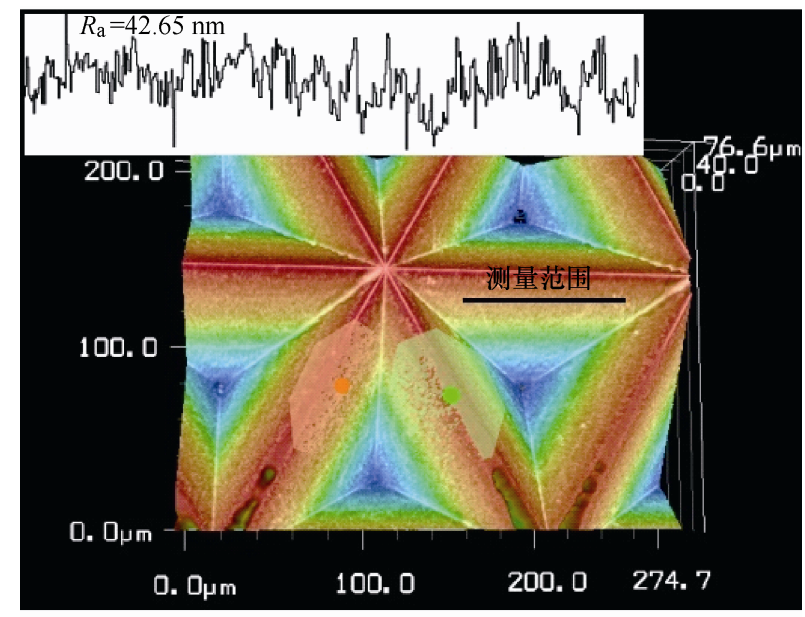

(c) 阴极超声振动 $(40 \mathrm{kHz}, 300 \mathrm{~W})$

图 2 电沉积表面形貌及粗糙度

从图 2 的粗粘度测试结果可以看出, 超声振动 表面粗粘度明显低于机械搅拌表面粗䊅度, 尤其是 超声功率为 $400 \mathrm{~W}$ 时。进一步对比铸层表面镍离子 结晶颗粒大小, 如图 3 所示, 超声振动的晶粒更小 且更均匀, 而机械搅拌的晶粒偏大且不均匀。

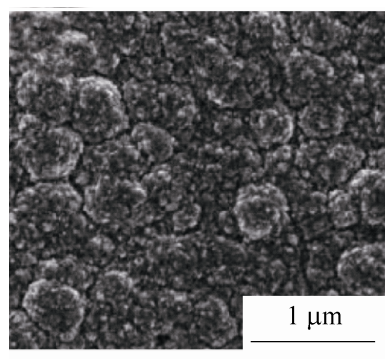

(a) 机械摚拌

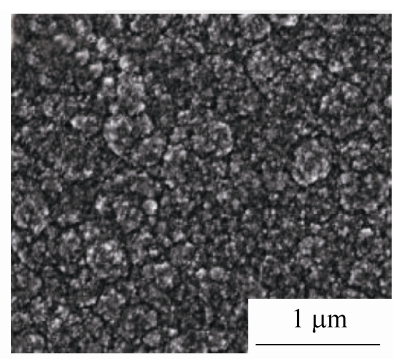

(b) 阴极超声振动
图 3 沉积表面 SEM 形貌

\section{2 机理分析}

(1) 阴极板的超声振动在电铸液边界层里产生 众多浴流，使电铸液均匀化。

通过对阴极板的振动模态计算可知, 第 30 阶固 有频率为 $40.03 \mathrm{kHz}$, 其模态如图 4 所示, 施加 $40 \mathrm{kHz}$ 的激励, 会将这个高阶固有振动模态激振起来 ${ }^{[19]}$ 。
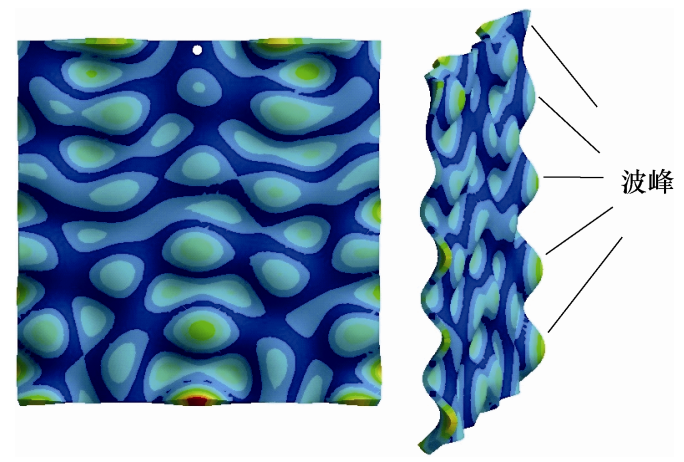

图 4 阴极镍板的振动模态 $(40 \mathrm{kHz})$
为了说明超声振动与机械搅拌对阴极附近电铸 液流动的影响, 对两个物理过程进行了仿真计算, 具体参数为: 超声振幅为 $10 \mu \mathrm{m}$, 频率为 $40 \mathrm{kHz}$, 机械摚拌速度为 $1000 \mathrm{r} / \mathrm{min}$ 。阴极板密度为 2050 $\mathrm{kg} / \mathrm{m}^{3}$, 弹性模量为 $1.9 \times 10^{11} \mathrm{~Pa}$, 电铸液密度为 $10^{3}$ $\mathrm{kg} / \mathrm{m}^{3}$, 黏度为 $10^{-3} \mathrm{~Pa} \cdot \mathrm{S}$ 。图 $5 \mathrm{a}$ 为超声振动电铸液 的速度场分布, 图 $5 b$ 为超声激励 $1 / 4$ 周期和 $3 / 4$ 周 期的流场局部放大图。和机械摚拌流场计算(图 6) 对比可以看出: 机械搅拌下, 电铸液在阴极板附近 的速度很低, 虽有流动, 但没有密集的浴流分布, 电铸液呈现二维流动状态; 而超声振动激励出阴极 板的弯曲振动模态, 其结果导致阴极板附近的电铸 液呈现众多三维小浴流，并且浴流的旋转方向以 40 $\mathrm{kHz}$ 的频率变化。

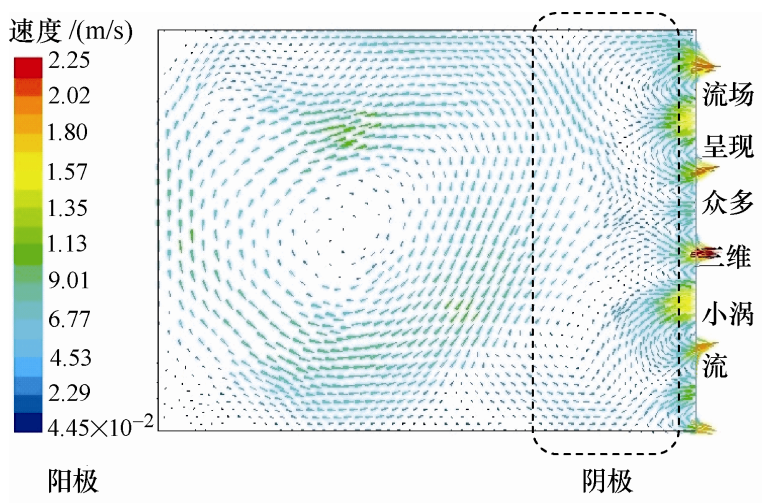

（a）电铸槽液速度场分布

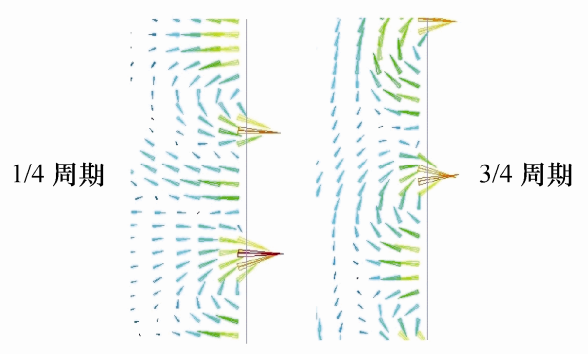

(b) 阴极附近速度场放大图

图 5 阴极超声振动下电铸液的速度场

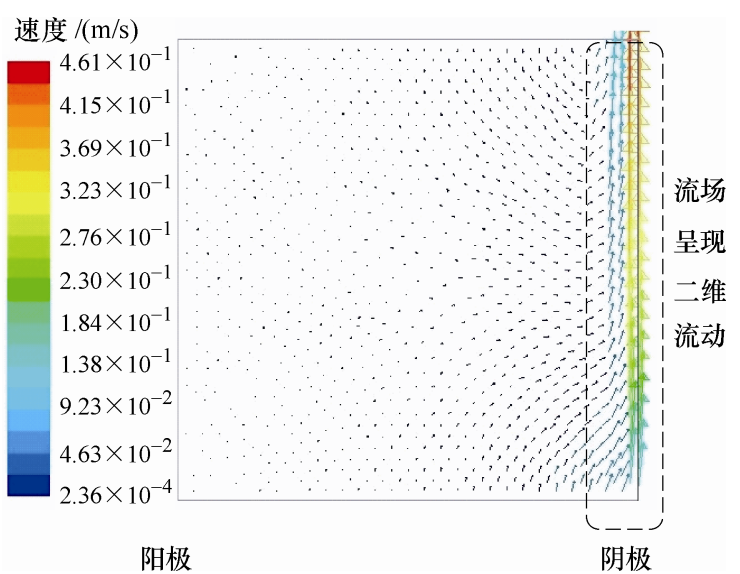

图 6 机械搅拌下电铸液的速度场 
显然, 就阴极板附近电铸液的混合效果而言, 超声振动的作用明显大于机械摚拌作用。在阴极板 高频振动作用下, 阴极板附近的电铸液被更充分地 搅拌，其镍离子浓度更接近于均匀化。

(2) 阴极板的的超声振动显著增大了镍离子在 电铸液里的有效扩散系数。

电铸液中的镍离子, 在阴极获得电子, 成为镍 原子结晶沉积; 在阳极上, 镍板表面的镍原子失去 电子, 成为镍离子进入电铸液。这个化学反应, 可 以写为

$$
\begin{aligned}
& \text { 阴极: } \mathrm{Ni}^{2+}+2 \mathrm{e}=\mathrm{Ni} \\
& \text { 阳极: } \mathrm{Ni}-2 \mathrm{e}=\mathrm{Ni}^{2+}
\end{aligned}
$$

在电铸液里面, 阴极表面是镍离子浓度最低的 地方, 阳极表面是镍离子浓度最高的地方, 这是因 为阴极是消耗镍离子的地方, 阳极是生产镍离子的 地方。因此, 从阳极到阴极, 存在着浓度梯度 ${ }^{[20]}$, 镍离子在阳极的浓度明显大于在阴极的浓度。

镍离子在电铸液里的输送, 由三部分叠加而成

$$
I=I_{\mathrm{e}}+I_{\mathrm{d}}+I_{\mathrm{c}}
$$

式中, $I_{\mathrm{e}}$ 为电场力引起的电流; $I_{\mathrm{d}}$ 为微粒子扩散引 起的电流; $I_{\mathrm{c}}$ 为电铸液宏观流动引起的电流。

其中, $I_{\mathrm{d}}$ 与镍离子有效扩散系数和浓度梯度有 关，受扩散方程控制

$$
I_{\mathrm{d}}=-D(\mathrm{~d} C / \mathrm{d} x)
$$

式中, $D$ 为镍离子在电铸液里的有效扩散系数; $\mathrm{d} C / \mathrm{d} x$ 为镍离子浓度梯度。

为了揭示超声振动对有效扩散系数的影响, 我 们计算了有效扩散系数。通过施加 $0 \mathrm{mV}$ 的负阶跃 电势, 可以测量其产生的电流对应时间的变化, 电 流和时间的关系服从 Cottre II 方程 ${ }^{[21-23]}$

$$
I_{\mathrm{d}}(t)=\left(n F A D^{1 / 2} c_{0}\right) /\left(c^{1 / 2} t^{1 / 2}\right)
$$

式中, $I_{\mathrm{d}}$ 为极限扩散电流; $n$ 为离子价数(对于本试 验的镍离子, $n=2) ; F$ 为法拉第常数; $A$ 为阴极板 的有效面积; $D$ 为镍离子的有效扩散系数; $c_{0}$ 为溶 液浓度; $c$ 为生长中心形状参数(根据 $\mathrm{SH}$ 理论模型, 生长中心为半球状, 取 $c=\pi) ; t$ 为时间。

对超声 $40 \mathrm{kHz}$ 辅助电铸和普通的机械摚拌辅 助电铸, $I_{\mathrm{d}}$ 随 $t^{1 / 2}$ 的变化曲线如图 7 所示, 有效扩 散系数可以根据 $I_{\mathrm{d}}-t^{1 / 2}$ 曲线的斜率来确定。本试验 中, 阴极板超声振动辅助电铸的有效扩散系数为 $3.71 \times 10^{-3} \mathrm{~cm}^{2} / \mathrm{s}$, 机械摚拌辅助电铸的有效扩散系数 为 $1.64 \times 10^{-4} \mathrm{~cm}^{2} / \mathrm{s}$ 。可见, 阴极超声振动相对于机械 摚拌, 能显著提高镍离子的有效扩散系数, 对边界
层镍离子的输送起到了促进作用, 这是传质得以强 化、浓差极化得以减弱、表面粗䊅度得以降低和电 流效率得以提高的一个重要前提。

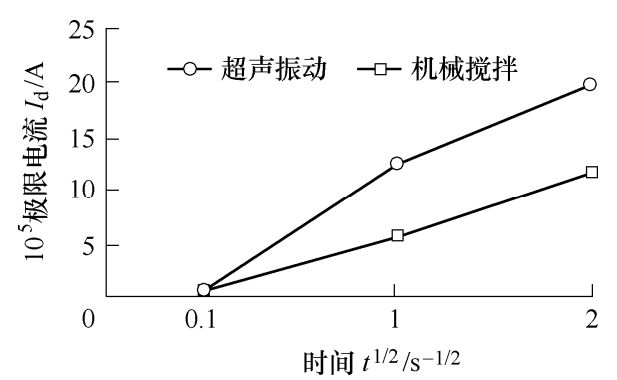

图 7 不同搅拌方式下的有效扩散系数

结合式(4)可知, 在同样的镍离子扩散电流 $I_{\mathrm{d}}$ 下, 即, 相同的沉积速率情况下, 超声振动电铸的 镍离子浓度梯度明显小于机械摚拌电铸的镍离子 浓度梯度。

(3) 均匀化后的镍离子浓度分布会抑制结晶 失稳。

垂直于阴极板表面的镍离子浓度梯度对电铸表 面粗䊁度的影响很大。镍离子浓度梯度越大, 越有 利于生长枝晶和肌瘤, 而且有利于生长尺寸大的晶 粒。垂直阴极表面方向的浓度梯度如图 8 所示: 假 如某个位置( $A$ 点)有刚刚生成的微小肌瘤, 这个肌瘤 头部位置的镍离子浓度大于没长肌瘤地方 $(B$ 点)的 镍离子浓度, 因此, 这会导致肌瘤头部的沉积速率 大一些。也就是说, 一旦有了肌瘤, 尽管它起初很 小, 但是, 它的生长速率会比没长肌瘤的地方大, 即结晶失稳, 它是肌瘤和枝晶生长的主要机理。因 为阴极板的超声振动能够带来镍离子浓度的均匀 化, 所以能够有效抑制结晶失稳, 减少肌瘤和枝晶 的形成和生长，从而降低表面粗䊁度。

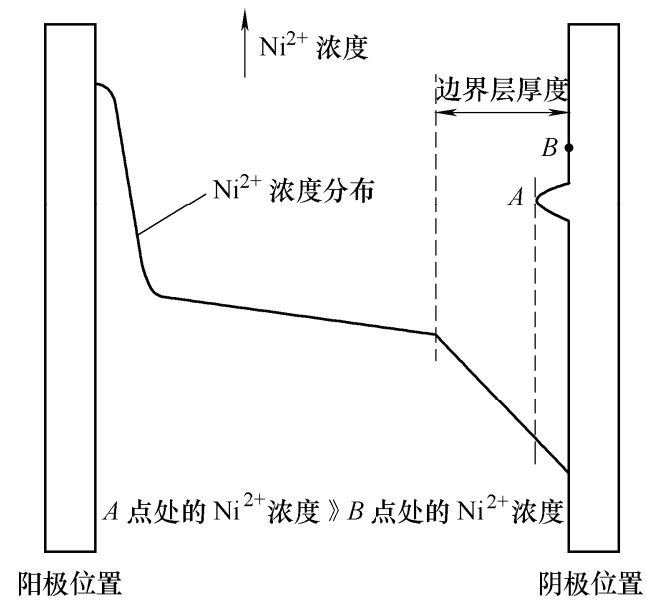

图 8 肌瘤结晶失稳示意图

（4）超声高频微射流作用抑制了氢气成核及析 出, 从而抑制了沉积层缺陷(氢气孔)。 
在阴极上, 不仅仅有镍离子的结晶沉积反应, 还伴随着氢气的析出: $2 \mathrm{H}^{+}+2 \mathrm{e}^{-}=\mathrm{H}_{2} \uparrow^{[1]}$ 。这个电化 学反应, 会在电铸表面形成气孔, 不利于表面质量。 氢离子获得电子的能力大于镍离子, 因此, 在理论 上, 电铸镍的同时很难抑制氢气析出。不过, 我们 的超声振动辅助电铸试验, 表明氢气析出得到了抑 制, 它的机理分析如下。

氢离子以及金属离子在阴极上获得电子被还 原, 形成沉积, 这均需要成核和生长过程。成核是 需要跨越一个能量壁垒(activation energy), 此过程难 度大于在成核后的位置上离子的继续析出。

阴极板的超声振动, 在周围溶液中产生的高压 和低压高频交变微射流作用会清除阴极板上的氢气 核, 从而有效抑制了沉积缺陷(氢气孔), 如图 $2 \mathrm{~b}$ 和 图 2c 所示, 这和文献[24-25]中超声产生高速微射流 有利于电极表面的气泡解吸, 清洁电极表面的结论 是一致的。

图 9 为超声振动 $(40 \mathrm{kHz}, 400 \mathrm{~W})$ 和机械搅拌的 沉积效率对比曲线: 试验开始 $1 \mathrm{~h}$ 内将电流密度逐 渐升高, $1 \mathrm{~h}$ 达到稳定电流密度 $5 \mathrm{~A} / \mathrm{dm}^{2}$, 共电铸 $5 \mathrm{~h}$, 对比超声振动和机械搅拌, 前者的沉积效率比后者 大 $52.2 \%$ 。

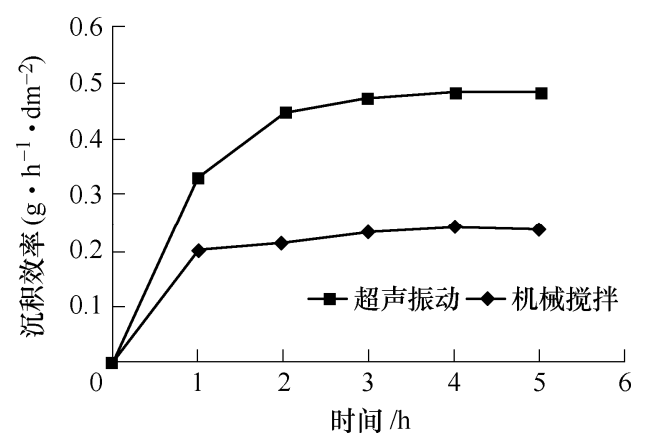

图 9 沉积效率

可见, 超声在电铸液中产生的高压和低压高频 交变微射流作用清除阴极板上的氢气，不仅有效抑 制了沉积缺陷(氢气孔), 也提高了电流效率, 这和 AMARA 等 ${ }^{[26]}$ 的研究结论一致。

\section{3 结论}

在电铸时, 对阴极板施加与板面垂直方向的超 声振动, 会激励出阴极板的高频弯曲振动模态, 此 高频振动模态能使阴极板附近的电铸液产生众多三 维小浴流, 这些高频变化的小浴流使得边界层溶液 充分混合, 这个混合导致阴极板附近的镍离子浓度 均匀化, 抑制了镍离子的结晶沉积失稳(肌瘤和枝 晶)。超声在电铸液中产生的高频交变微射流会清除
阴极板上的氢气核, 可以抑制电铸层的沉积缺陷(氢 气孔), 并减小阴极氢离子的析出反应速率, 改善了 电流效率。

\section{参 考 文 献}

[1] 陈钧武, 何士恒. 电铸原理与工艺[M]. 北京: 化学工 业出版社, 2010.

CHEN Junwu, HE Shiheng. Principle and process of electroforming[M]. Beijing: Chemical Industry Press, 2010.

[2] 刘冲, 李苗苗, 施维枝, 等. 基于 SU-8 厚胶光刻工艺 的微电铸铸层尺寸精度控制新方法 [J]. 机械工程学报, 2011，47(3): 179-185.

LIU Chong, LI Miaomiao, SHI Weizhi, et al. New method for dimensional precision control of electroformed parts by using micro electroforming technique with SU-8 Thick photoresist[J]. Journal of Mechanical Engineering, 2011, 47(3): 179-185.

[3] MiCHAEL B, THOMAS S, BOGUSLAW $\mathrm{W}$, et al. Highly precise micro-retroreflector array fabricated by the LIGA process and its application as tapped delay line filter [J]. Applied Optics， 2012， 51(25): 5995-5999.

[4] 杨光, 皮钧. 反光膜微结构阵列电铸电流密度分析 [J]. 电镀与环保, 2014(3): 22-26.

YANG Guang, PI Jun. An analysis of density distribution in microstructure array electroforming of reflective membrane[J]. Electroplating \& Pollution Control, 2014(3): 22-26.

[5] DU L Q, YANG T, ZHAO M, et al. Study on improving thickness uniformity of microfluidic chip mold in the electroforming process[J]. Micromachines, 2016, $7(1): 7-12$.

[6] LI J, ZHANG P, WU Y, et al. Uniformity study of nickel thin-film microstructure deposited by electroplating[J]. Microsystem Technologies, 2009, 15, 505-510.

[7] 杨光, 叶飞. 间接超声下电铸液温升及微棱镜镍层反光 性能[J]. 功能材料, 2014, 43(8): 883-888.

YANG Guang, YE Fei. Temperature rise and reflective performance of nickel films with microprism structure electrodeposited atindirect-ultraconic electrochemical deposition[J]. Journal of Functional Materials, 2014, 43(8): $883-888$

[8] ZHAO Z, DU L Q, TAO Y S, et al. Enhancing the adhesion strength of micro electroforming layer by ultrasonic agitation method and the application[J]. Ultrasonics Sonochemistry, 2016, 33(4): 21-25.

[9] MING P M, ZHU D, HU Y Y, et al. Experimental 
research on microelectroforming with ultrasonic agitation[J]. Key Engineering Materials, 2008, 375, 253-257.

[10] BECKETT M A, HUA I. Impact of ultrasonic frequency on aqueous sonoluminescence and sonochemistry[J]. Journal of Physical Chemistry A, 2001, 105, 3796-3802.

[11] LIU W Q, LEI W N, WANG C Y, et al. Ni-SiC nanocomposites electroplating process under ultrasonic and agitation[J]. Integrated Ferroelectrics, 2015, 167(1): 192-198.

[12] 明平美, 朱荻, 胡洋洋, 等. 超声微细电铸试验研究 [J]. 中国机械工程，2008，19(6): 644-647.

MING Pingmei, ZHU Di, HU Yangyang, et al. Experimental research on microelectroforming with ultrasound stirring $[\mathrm{J}]$. China Mechanical Engineering, 2008, 19(6): 644-647.

[13] 翟言强, 李克智, 李贺军, 等. 超声电沉积碳/碳复合 材料磷灰石涂层的工艺优化 [J]. 材料工程, 2007(4): 27-31.

ZHAI Yanqiang, LI Kezhi, LI Hejun, et al. Optimization of process parameters of calcium phos phate coating on $\mathrm{C} / \mathrm{C}$ composites by sonoelectrode position technique[J]. Journal of Materials Engineering, 2007(4): 27-31.

[14] 吴蒙华, 傅欣欣, 李智, 等. 超声电沉积镍/纳米碳化 硅复合镀层组织结构研究 [J]. 机械工程材料, 2004, 28(12): $46-48$.

WU Menghua, FU Xinxin, LI Zhi, et al. Microstructure of ultrasonic-electrode positing $\mathrm{Ni} / \mathrm{Nano}-\mathrm{SiC}$ composite coatings[J]. Materials for Mechanical Engineering, 2004, 28(12): $46-48$.

[15] DALAS E. The effect of ultrasonic field on calcium carbonate scale formation[J]. Journal of Crystal Growth, 2001, 222(1): 287-292.

[16] 夏法锋, 吴蒙华, 贾振元, 等. 超声波对纳米 Ni-TiN 复合镀层的影响 [J]. 功能材料, 2008，39(4): 690-691. XIA Fafeng, WU Menghua, JIA Zhenyuan, et al. Effect of ultrasonic wave on nano Ni-TiN composite coatings[J]. Journal of Functional Materials, 2008，39(4): 690-691.

[17] 杨艳玲, 申勇峰, 陈进耿, 等. 超声波摚拌-脉冲电沉 积法制备纳米镍[J]. 金属学报, 2007, 43(8): 883-888. YANG Yanling, SHEN Yongfeng, CHEN Jingeng, et al. Nanocrystallne nickelcoating prepared by pulsed electrodeposition combined with ultrasonic agitation[J]. Acta Metallurgica Sinica, 2007，43(8): 883-888.

[18] 刘剑飞. 超声改善微电铸铸层均匀性研究 $[D]$. 大连: 大 连理工大学, 2013.
LIU Jianfei. Improvement of thickness uniformity of micro-electroformed layers by ultrasonic agitation[D]. Dalian: Dalian University of Technology, 2013.

[19] 胡海昌. 多自由度结构固有振动理论 [M]. 北京: 科学 出版社, 1987.

HU Haichang. The theory of natural vibration of multi-degree freedom structure[M]. Beijing: Science Press, 1987.

[20] 李荻. 电化学原理 [M]. 北京: 北京航空航天大学出版 社, 1998 .

LI Di. Electrochemical principle[M]. Beijing: Press of Beijing University of Aeronautics and Astronautics, 1998.

[21] SCHARIFKER , B , HILLS , G. Theoretical and experimental studies of multiple nucleation[J]. Electrochimca Acta 1983, 28 (7), 879-889.

[22] BARD A J, FAULKNER L R. Electrochemical methods fundamentals and applications[M]. New York: Press of John Wiley \& Sons, 1980.

[23] 吴小英, 杨丽坤, 间慧, 等. $\mathrm{n}$ 型半导体硅电极表面 $\mathrm{Au}$ 的电化学成核机理 [J]. 物理化学学报, 2015, 31(9): 1708-1714.

WU Xiaoying, YANG Likun, YAN Hui, et al. Electrochemical nucleation of $\mathrm{Au}$ on n-type semiconductor silicon electrode surface[J]. Acta Physico-Chimica Sinica, 2015, 31(9), 1708-1714.

[24] 冯若, 李化茂. 声化学及其应用 $[\mathrm{M}]$. 合肥：安徽科技 出版社, 1992.

FENG Ruo, LI Huamao. Sonochemistry and its aplications[M]. Hefei : Anhui Science \& Technology Press, 1992.

[25] SUSLICK K S. Sonochemistry[J]. Science， 1990，247: 1439-1445.

[26] AMARA N, RATSIMBA B, WILHELM A, et al. Growth rate of potash alum crystals: Comparison of silent and ultrasonic conditions $[\mathrm{J}]$. Ultrasonics Sonochemistry, 2004, 11(1): 17-21.

作者简介：杨光(通信作者), 女, 1971 年出生, 博士，教授。主要研究 方向为精密加工、电化学加工。

E-mail: yangg@jmu.edu.cn

胡志超, 男, 1975 年出生, 副教授。主要研究方向为电化学加工。

E-mail: hzc0204@163.com

张军, 男, 1966 年出生, 博士, 副教授。主要研究方向为流体力学。

E-mail: bul10202@sina.com

皮钧, 男, 1962 年出生, 博士, 教授。主要研究方向为精密加工、超声 加工技术。

E-mail: pijun@jmu.edu.cn

刘中生, 男, 1960 年出生, 博士, 教授。主要研究方向为机械动力学和 精密加工。

E-mail: liuzhongsheng@jmu.edu.cn 